\title{
Science in Development*
}

\author{
By the Right Hon. Lord Rutherford, O.M., F.R.S.
}

$\mathrm{I}^{\mathrm{x}}$ surveying the great tide of advance in the physical sciences during the past forty years, it is not always recognized how much the progress of discovery has been influenced, and indeed in many cases controlled, by the improvement of laboratory technique and by the development of new instruments and methods of measurement.

To appreciate the rapid changes which have occurred, it is probably simplest to contrast the laboratory equipment of to-day with that available at some well-defined epoch in the past. I choose for this purpose the year 1895, that turning point in the history of physics, which saw the discovery of X-rays, to be followed in quick succession by the diseoveries of radioactivity and of the electron. This choice of year seems to me convenient as it marks a natural dividing line between the older and the newer physics.

First of all, I should like to refer to the great advantages to research in general that have followed from the invention of an efficient form of lead accumulator. Before the commercial manufacture of the accumulator, the experimenter had to rely on primary batteries for the supply of electric current in his investigations. The Daniell cell or corresponding battery was used for small currents-as, for example, in comparing resistances by the Wheatstone bridge. For the supply of currents of the order of an ampere or more, Grove's cells were often used, but these had only a working life of a few hours and had to be freshly prepared each day. When I first began some investigations on the magnetic properties of iron in 1893 at Canterbury College, Christchurch, New Zealand, I found it necessary to prepare each morning a battery of about a dozen Grove cells. This involved the cleaning and amalgamation of the zinc plates and adding the necessary acids. Fortunately, large platinum plates for the electrodes were available. I found this battery of low internal resistance a very convenient means of obtaining substantial and steady currents, but after several hours' work the battery showed obvious signs of exhaustion and accurate work with it was impossible.

In order to illustrate the provision for research forty years ago, I shall take as an example the Cavendish Laboratory, which was directed by Sir J. J. Thomson, who was then on the verge of beginning his famous experiments on the electrical

* From the twelfth annual Norman Lockyer Lecture of the British Science Guild, delivered on November 12. conductivity in gases produced by $\mathrm{X}$-rays and his investigations of the nature of the cathode rays. The equipment of the Cavendish Laboratory both for teaching and research was probably typical of the better European physical laboratories of that time. Largely due to the labours of the late Sir Richard Glazebrook and of Sir William Napier Shaw, a well-organized system of laboratory instruction had been arranged for students, and this was extended and elaborated through the years by that genius in the design of laboratory apparatus for teaching purposes, Dr. G. F. C. Searle.

While the provision for teaching and research was in general excellent in the older sciences of optics, heat, sound and properties of matter, in contrast with to-day the amount of electrical apparatus, particularly on the measuring side, was sparse and in some cases almost non-existent. Apart from reflecting galvanometers of the moving magnet and moving coil types and a tangent galvanometer, there was no portable instrument for measuring current except one very crude form of ammeter. The only portable method of measuring voltage was a rather ponderous Cardew voltmeter of the hot wire type, which was of very limited utility and accuracy. It was not until a few years later that those admirable instruments for measuring current and voltage designed by the late Mr. Edward Weston of the United States came into laboratory use. To-day, hundreds of measuring instruments of this or similar types are required in electrical research, and indeed a single experimenter may feel himself hardly used if a dozen of these instruments are not available, with appropriate wire rheostats to control the voltages and current.

Apparatus for work in electrostatics was more plentiful, including a number of types of quadrant electrometer, which, however, were usually both unreliable and capricious instruments. The quadrants were large and the needle unnecessarily heavy. The moving system was often supported by a silk fibre and the position of the needle controlled by a small magnet attached to it. A platinum wire was attached to the bottom of the needle and dipped in strong sulphuric acid, which formed the inner coating of a small glass condenser. In order to obtain a reasonable sensitivity, the needle and condenser required to be charged to a fairly high potential. This was done by charging the jar with 
a spark or two from an electrophorus or from a charged Leyden jar. Unless very special precautions were taken with the insulation, the charge on the condenser leaked away rapidly and the sensitivity of the electrometer varied markedly from hour to hour, while the zero of the needle was very unsteady. Under these conditions, it was difficult to make observations over any but a short period with any accuracy. Lord Kelvin, the pioneer in the construction of quadrant electrometers, had designed a more elaborate and more serviceable instrument in which the potential of the charge given to the needle could be brought to a definite value by the use of an attracted disk electrometer incorporated with the instrument. One of these electrometers, which was available in the Laboratory, was made after great effort to work satisfactorily by Prof. J. S. Townsend, and was used by him in his well-known researches on the electrification of gases and on the properties of ions in gases. This, I believe, was the first time this instrument, which was of moderate sensitivity, had been used in systematic researches of importance.

A great advance was made about 1900 when Dozalek designed a quadrant electrometer with a fine quartz suspension and a very light needle, which gave a good sensitivity when the needle was charged to the comparatively low potential of a few hundred volts.

In place of the quadrant electrometer, the tilted electroscope designed by C. T. R. Wilson in 1903 was often used. This had a low capacity and a voltage sensitivity comparable with an electrometer. Recently a very sensitive form of this instrument has been designed by $\mathrm{H}$. Carmichael and used for experiments on the bursts of ionization produced by the cosmic rays. After the Great War, still more sensitive electrometers were designed; for example, a modified quadrant by Compton, and a binant electrometer of high sensitivity by Hofmann, while a robust and portable type of string electrometer of good sensitivity designed by Lindemann has proved very serviceable in many directions. At this stage, I must not omit to mention the extraordinary utility of a modified form of the gold leaf electroscope for measurement of ionization, particularly in the study of the radioactive bodies. This instrument, which had been used for detecting charge for a century or more, was developed into an accurate measuring instru. ment largely due to the modifications introduced by C. T. R. Wilson. In course of time the electroscope displaced the quadrant electrometer for measuring the periods of decay of radioactive bodies and for a study of the $\alpha$-, $\beta$ - and $\gamma$-rays.

The changes in forms of apparatus so far mentioned did not involve any essential new principle, but were rather the result of improvement in design to increase their sensitivity and reliability and to adapt the form of the instrument to meet the needs of investigators. To this period, however, belongs the development of an instrument which to my mind is the most original and wonderful in scientific history-I refer to the 'cloud' or 'expansion' chamber of C. T. R. Wilson. I had the good fortune to be present in 1895 in the Cavendish Laboratory when Wilson was examining the formation of water drops in damp dust-free air by sudden adiabatic expansion of the gas. When $\mathrm{X}$-rays were discovered, Wilson at once showed that the ions produced by the X-rays in their passage through the gas acted as nuclei for the condensation of water upon them under certain conditions of supersaturation. A cloud of visible water drops was formed and in a sense each ion was rendered visible by the water drop formed round it.

The next important advance was made in 1912, when Wilson devised the modern type of expansion chamber and showed that the tracks of individual $\alpha$-particles and fast electrons are rendered visible by the trail of water drops formed on the ions liberated by the passage of these flying particles through the gas. It was a wonderful advance to be able to see, so to speak, the details of the adventures of these particles in their flight through the gas. Anyone with imagination who has seen the beautiful stereoscopic photographs of the trails of swift $\alpha$-particles, protons or electrons, cannot but marvel at the perfection of detail with which their short but strenuous lives are recorded. The cloud chamber has proved an invaluable aid to research in many directions and has become allimportant in recent work on artificial transmutation. This instrument provides in a sense a final court of appeal in which the experimenter may place his trust. I cannot imagine anyone with the most vivid scientific imagination who could have predicted the possibility of an instrument endowed with such unique powers and potentialities.

\section{HIGH VACUA}

Of all changes in laboratory apparatus and technique in the period under review, none has been more revolutionary in type or of more importance to the progress of science and industry in many directions than the improvements in methods of rapid production of high vacua. Vacuum research has a long history and has persistently engaged the attention of scientific men from the year 1643, when Torricelli, the assistant of Galileo, made the first mercury barometer. The first mechanical air pump was devised by von Guericke in 1650 . It was a simple type of suction pump 
with a well-fitting plunger and automatic leather valves, and was probably able to reduce the pressure of air in a sealed container to between 1/100 and $1 / 1,000$ of an atmosphere.

While mechanical pumps were improved, no great advance in principle or practice of producing high vacua took place for nearly two hundred years. To be sure, many investigations were made, for example, by Hawksbee, to investigate the beautiful glow discharges observed when an electrical spark passed through a vessel containing gas at very low pressure. While mechanical pumps were often used for special purposes, the Torricelli principle was more popular and effective in producing a good vacuum.

The most important stage in the subsequent advance was the invention in 1855 of the mercury pump proper by Geissler, who used it for exhausting discharge tubes in order to illustrate the striking luminous effects which arise when the electric discharge from an induction coil is passed through the low-pressure gas contained in the tube. Such Geissler tubes were long used for illustrations in lectures, and replicas of them may still be seen to-day. The invention of the mercury pump gave the possibility of producing a really good vacuum by long-continued pumping. The advantage over the mechanical pump was due primarily to the elimination of the mechanical valve and the dead space. The mercury pump was used by Hittorf for studying the marked changes in the appearance of the electric discharge as the pressure of gas was decreased to the lowest value obtainable. $\mathrm{He}$ observed the conditions of production of the now well-known cathode rays and was, I think, the first experimenter to appreciate that, in order to maintain a permanently good vacuum in a sealed tube, it was necessary to 'degas' the walls of the tube and electrodes by frequent heating and longcontinued bombardment by the electric discharge. In this way he produced, for the first time, a tube with so good a vacuum that an induction coil failed to produce any visible discharge through the highly rarefied gas.

The work of Hittorf was extended and improved upon by Sir William Crookes, who used a modified form of mercury pump invented by Sprengel in 1865 , which could readily be made automatic in action. A number of modifications of Geissler's original pump were made from time to time, and of these the pump devised by Toepler in 1862 came into general use towards the end of the nineteenth century. When I first came to the Cavendish Laboratory in 1895, modified forms of Toepler pump, made by that skilled glassblower $\mathrm{Mr}$. Everett, were in general use. One of these pumps was used by Sir J. J. Thomson in his famous experiments which proved the nature of the cathode rays. In those days, it was usual to employ a one- or two-cylinder Fleuss pump for preliminary pumping of the vessel to a pressure below $1 \mathrm{~mm}$. This pump was a greatly improved form of the original mechanical air pump of von Guericke. The moving piston and working parts were covered with a layer of oil. The small quantity of air entrapped by the upward motion of the piston was forced with the oil through a spring valve. By these modifications, the efficiency of the pump in reaching a low pressure was much increased. Under good conditions a vacuum as low as $1 / 50 \mathrm{~mm}$. could be obtained. It should be borne in mind that the production of a good cathode ray vacuum in a discharge tube in the old days was a lengthy and laborious process with the slow-acting Toepler or Sprengel pumps. Several hours pumping was required to produce a good vacuum by these methods, while to-day, with our fast diffusion pumps, a similar result can be obtained in a few minutes or even seconds. It is of interest to record that the X-ray tubes first used for radiographic purposes were all painfully exhausted by one of these early forms of mercury pump.

From the point of view of ease and simplicity in maintaining a good vacuum, the discovery of Sir James Dewar in 1904 that specially prepared charcoal was a strong absorbent of gases at low temperature has proved of great importance to science. The tube to be exhausted was connected with a side tube containing charcoal. After preliminary exhaustion with a mechanical air pump, the charcoal was immersed in liquid air, when the residual gases in the tube were rapidly absorbed by the charcoal and a high vacuum produced which could be maintained for hours. This method of producing a controllable degree of vacuum was widely used for many years in our laboratories. For example, this method was employed by Sir J. J. Thomson in his well-known experiments on the positive rays, and on account of its simplicity is still used by Aston in his investigations with the mass-spectrograph.

The last thirty years have seen great changes in our methods of rapid production of high vacua, and many new and ingenious types of fast pumps have been invented. This rapid development has been in large measure due to the remarkable powers of invention shown by Dr. Gaede, who has designed a whole series of pumps of great scientific and technical interest.

The first glass diffusion pump employing a stream of mercury vapour was described by him in 1915, and has been followed by a succession of designs of increasing efficiency. This pump, which has the advantage of containing no movable parts, can be constructed either of glass or metal, and a great variety of forms have been used, 
particularly in the case of small glass pumps for ordinary laboratory use. Improvements in the design of the all-metal diffusion pump to ensure higher pumping speeds were early made by Langmuir in the United States and by Kaye in Great Britain. Although there are great varieties of these pumps both as regards size, form and construction, they all depend on the same general principle. The gas to be removed diffuses through a suitable aperture into a stream of hot mercury vapour which carries the gas away with it, the mercury vapour being afterwards condensed and the gas removed by a backing pump. The speed of exhaustion of these pumps is astoundingly fast, and theoretically there is no limit to the degree of vacuum that can be obtained. In general, a single-stage pump requires the pressure to be reduced by a fore pump from $1 / 10$ to $1 / 100 \mathrm{~mm}$. before the pump comes into action. Gaede has designed a 3-stage all-steel pump which begins to operate at a pressure of $20 \mathrm{~mm}$. and has a high speed of exhaustion.

In the mercury diffusion pumps it is usual to employ a liquid air trap to prevent the back diffusion of the mercury vapour into the vessel to be exhausted. This is a disadvantage both from the point of view of economics and of efficiency in pumping, and has been got over by replacing mercury by a special oil which has a negligibly small vapour pressure at ordinary temperatures, so that liquid air is dispensed with. The first oil diffusion pump was constructed in Great Britain by Mr.C.R.Burch of the Metropolitan-VickersResearch Laboratory, using special oil obtained by molecular distillation. These pumps are very efficient and are very widely used, particularly for large sizes of pump where great volumes of gas have to be rapidly removed.

The invention and development of the diffusion pump has given to science a new and extraordinarily effective instrument for the rapid attainment of the highest degree of vacuum. Not only has its use greatly simplified investigations in very high vacua, but also it has made possible many new technical developments. With the aid of these fast pumps, we can maintain large vessels at such a low pressure, even when gases are pouring in through a small opening, that voltages of the order of 1 million volts can be applied without the passage of a discharge. This has made it possible to obtain streams of very fast particles, for example, protons and deuterons, which have proved so effective in studying the transmutation of the elements.

I have already mentioned incidentally some of the more fundamental discoveries that have resulted from a study of the electric discharge through rarefied gases; for example, the production and nature of the cathode rays, the discovery of the $\mathrm{X}$-rays and the development of new methods of analysis of the charged atoms and molecules produced in the discharge tube. The resolving power of this method of chemical analysis has been so improved by Aston that it has been found possible not only to show the isotopic constitution of many of the ordinary elements, but also to determine the relative masses of the atoms with high precision. The use of high vacua led to the investigation by $\mathrm{O}$. W. Richardson of the copious emission of electrons from incandescent bodiesa discovery from which has originated many important industrial applications. In general, the study of processes in very high vacuahasadded much to our knowledge in many directions. The pioneer work of Langmuir on chemical reactions at very low pressures and on adsorption phenomena has opened a new and important field of inquiry which is still being developed. Much light has been thrown on the formation of monomolecular films on surfaces and their importance in catalytic reactions, and in general on the nature of chemical and physical forces which come into play. The use of molecular rays by Stern and Gerlach was only made possible by high vacuum technique.

I have so far only spoken of methods of producing high vacua, but in the course of this development not only have reliable methods been devised for measurements in situ of the residual pressure of the gas, but also great advances have been made in industrial laboratories in the technique of making metal seals with glass, for example, copper, steel and tungsten. This has made possible the production on a commercial scale of robust tubes of combined glass and metal such as are used for high-power rectifiers and oscillators. In addition, the industrial laboratories have been responsible for many improvements in technique. Many new kinds of glass have been produced and methods devised for graded glass joints, such as pyrex to soft glass and silica to pyrex. The high-frequency eddy current heater for degassing metal parts in situ has proved of great value, while the technique for degassing tubes, whether by heat or the electrical discharge or both together, has been highly developed. Special machines have been devised for rapid exhaustion and sealing of glass bulbs. The production of low vapour pressure oils and greases has made possible the efficient and economical oil diffusion pump and improved methods for air-tight joints.

In the development of high vacua, the mutual reaction between science and industry is illustrated in a striking way. The pioneer work of the physicist led to the development of methods of producing high vacua and to many new discoveries of the 
properties of matter in rarefied gases. These new methods have been applied to industry in a great variety of ways, and in turn have provided the physicist not only with improved forms of apparatus but also with many new technical devices which have made possible still further progress in pure physics. For example, the production on a commercial scale of efficient amplifiers, thyratrons and rectifiers, has provided the physicist with new and powerful weapons for extending his investigations in many directions. These developments have made possible not only the production of streams of swift particles for work on transmutation, but also methods of automatic counting of the swift particles which result from the transformations. These new counting methods owe much to the ingenuity of Dr. Wynn-Williams.
On account of these great improvements in technique and methods, progress in our knowledge has been so accelerated in the last few years that it is not easy for the investigator to keep in close touch with the rapidity of advance of new knowledge.

There have also followed great improvements in the efficient production of X-rays, and I must not omit to mention the development by Coolidge of the hot cathode, highly exhausted X-ray tube, known by his name, which has proved so valuable both for scientific and medical purposes. I could give many other illustrations of the beneficial effects of this close mutual relation between the workers in our university and technical laboratories -a happy relation which cannot fail to be of good augury for the future.

\title{
J. L. Macadam: Father of Modern Road-Making
}

\author{
By Dr. R. Quarendon
}

$T^{\top}$ HE centenary of the death of J. L. Macadam comes at a particularly fitting time, because the history of the roads is passing through a stage very similar to that which marked the years of Macadam's greatest activity. Then, as now, transport was being handicapped by the lack of modern highways, and the march of progress hindered by out-of-date conditions.

The roads were in many cases little better than they had been in the Middle Ages. Two hundred years of neglect, ignorance and prejudice had left them in an appalling state of disrepair. Journeys which now take hours lasted for days or even weeks, and were undertaken only after anxious consideration.

Parliament had done its best to suppress the wheeled vehicle, or to regulate it as a nuisance. Act after Act was passed restricting the loads to be carried, the number of horses per wagon, and the size and shape of the wheels. Excepting the 22,000 miles of turnpikes, the repair of the roads was in the hands of the parishes, who were empowered to conscript labour-known as 'statute labour'-whose services were given with the greatest unwillingness. The 1,100 turnpike trusts were run in many cases by ignorant and incompetent men who took the revenues but spent little on repairs.

Lacking engineering training, Macadam seems to have developed his method from first principles. It was at thirty years of age, after having gained a fortune as an agent for the sale of prizes in the
American War of Independence, that he returned to Scotland to begin his experiments. As magistrate, deputy lieutenant and road trustee for Ayrshire, he was able to give considerable time to this work. His efforts met with the greatest opposition, in spite of the fact that it was his own money which was being spent on the investigation. The Scots people did not want a hard metalled road. They preferred to drive their cattle and geese to market over the soft earth roads which then prevailed.

For many years, Macadam was unable to test his ideas on a suitable scale. In 1798 he was appointed agent for revictualling the $\mathrm{Navy}$ in the western ports, and his duties afforded little time for road research. It was not until 1815, when he became surveyor-general of the Bristol roads, that the opportunity for which he had been waiting arrived.

The usual method of making a road in those days was to dig earth from ditches cut along the side, and to pile it in the middle, in the pious hope that the traffic would beat it into a hard surface. Sometimes gravel was thrown on top, but in wet weather the earth and gravel soon became churned into a pebbly mud. Although he was a pioneer of his day, all Macadam really did was to provide a durable, properly drained bed of road metal. He excavated the earth to a depth of 6-10 inches, according to the nature of the subsoil, and filled in the trench with layers of stone broken to one-inch cubes. The traffic 